JOURNAL

of Health Inequalities

\title{
Gender differences in smoking-related diseases
}

\author{
Eva Negri \\ University of Milan, Italy
}

ADDRESS FOR CORRESPONDENCE: Dr. Eva Negri, University of Milan, Via Gian Battista Grassi 74, Italy, e-mail: eva.negri@unimi.it

Supplementary materials (slides) are available in Webappendix 12 at the journal's website: https://www.termedia.pl/Journal/Journal_of_ Health_Inequalities-100.

The prevalence of smoking varies greatly by gender, time period, and geographic area. In high-income countries, albeit with substantial time differences, the prevalence of male smokers has increased dramatically in the last century, reaching a peak between 1950 and 1980, and decreased thereafter. Tobacco smoking started to increase in women a few decades later than in men, and declines have started only more recently. Smoking prevalence is rapidly increasing in men in many low and middle countries, but not in women.

"Smoke like a man, die like a man" is the title of a review on sex, gender, and lung cancer, which summarises in a nutshell the health consequences of smoking in women, concerning diseases common to the two sexes. Epidemiological evidence clearly shows that women are at least as susceptible as men to lung and other smoking-related cancers, respiratory diseases, cerebro- and cardiovascular diseases, and all other health problems caused by smoking. The Million Women Study, a prospective study including 1.3 million UK women, showed that (in women aged 50-79 years) overall mortality of smoking women was three times higher than in never smokers, and that smokers lose at least 10 years of lifespan. These results are similar to those found in men.

As in their male counterparts, stopping smoking is an effective way to avoid most of the damage caused by smoking, and even cessation at 50 years of age avoids at least two thirds of the excess mortality seen in women that continue smoking.

Squamous cell carcinoma of the cervix has also been shown to be increased in smokers. Smoking does not appear to be associated with breast or ovarian cancer, while an inverse association has often been reported for endometrial cancer, stronger in postmenopausal women. Some studies suggest that smoking may increase the risk of dysmenorrhea, secondary amenorrhea, and men- strual irregularity. Women who smoke have a younger age at menopause and more menopausal symptoms, and may have a decreased risk of uterine fibroids.

All stages of the reproductive functions are affected by smoking, and the effects are dose-dependent. Smoking is positively associated with several adverse pregnancy outcomes, including infertility, ectopic pregnancy, placental abruption, miscarriage, preterm birth and low birthweight, and perinatal mortality.

The tobacco industry has developed specific marketing strategies for different population segments, including women, including marketing cigarette brands specifically directed to women. Tobacco advertising dedicated to women has associated cigarette use with independence, modernity, glamour, stress release, and weight control. These strategies have been used successfully in high-income countries and are being replicated and adapted in low- and middle-income countries.

Smoking cessation programs targeting women have been developed, addressing their specific needs. Interventions to promote smoking cessation during pregnancy have been shown to reduce not only smoking in late pregnancy, but also low birthweight and preterm births, although there was a tendency to restart smoking after delivery.

Key words: tobacco smoking, gender differences, health effects.

\section{DISCLOSURE}

The author reports no conflict of interest. 